[Article]

\title{
$\mathrm{Y}-\mathrm{MnO}_{2}$ 的形貌控制及其甲苯液相氧化性能
}

\author{
林 健 蔡 钒 张国玉 杨乐夫 ${ }^{*}$ 杨金玉 方维平 ${ }^{*}$ \\ (厦门大学化学化工学院, 醇梄酯清洁生产国家工程实验室, 福建厦门 361005)
}

\begin{abstract}
摘要：通过在回流法制备流程中引入CTAB(十六烷基三甲基溴化铵)、PEG6000(聚乙二醇 6000)及 P123(聚 环氧乙烷-聚环氧丙烷-聚环氧乙烷三嵌段共聚物)等表面活性剂对 $\gamma-\mathrm{MnO}_{2}$ 催化剂进行形貌控制, 同时采用 $\mathrm{X}$ 射 线衍射 $(X R D)$ 、扫描电镜 $(S E M) 、 N_{2}$ 吸附 $(B E T)$ 、热重分析 $(T G A) 、 O_{2}$ 程序升温脱附 $\left(\mathrm{O}_{2}-T P D\right)$ 以及 $\mathrm{H}_{2}$ 程序升温还 原 $\left(\mathrm{H}_{2}-\mathrm{TPR}\right)$ 等技术对不同形貌 $\gamma-\mathrm{MnO}_{2}$ 的结构、氧脱附及还原性能进行表征, 并考察了其在常压和无溶剂条件 下甲苯选择性氧化反应体系的催化特性. 同时, 对于陈化时间对形貌的影响作用进行了考察. 结果表明: 不同形 貌的 $\gamma-\mathrm{MnO}_{2}$ 的氧化还原特征及催化活性存在显著差异, 其中在经 PEG6000 进行修饰的 $\gamma-\mathrm{MnO}_{2}$ 中含有较多的 阴离子空位及混合价态, 因此有助于分子氧在表面的活化, 具有较高的表面比活性; 而经P123进行表面修饰的 $\gamma-\mathrm{MnO}_{2}$ 成晶结构规整、比表面积大, 对甲苯液相直接氧化反应则表现出最佳的质量比活性, 甲苯转化率达 $18.1 \%$, 含氧化合物总选择性为 $87.4 \%$, 其中苯甲酸的选择性达到 $73.2 \%$.
\end{abstract}

关键词: $\gamma-\mathrm{MnO}_{2} ;$ 甲苯选择性氧化; 形貌控制; 氧物种; 表面活性剂 中图分类号: 0643

\section{Preparation of Morphology-Tuned $y-\mathrm{MnO}_{2}$ and Catalytic Performance for the Liquid-Phase Oxidation of Toluene}

\author{
LIN Jian $\quad$ CAI Fan $\quad$ ZHANG Guo-Yu YANG Le-Fu* YANG Jin-Yu FANG Wei-Ping* \\ (National Engineering Laboratory for Green Chemical Productions of Alcohols, Ethers, Esters, College of Chemistry and Chemical \\ Engineering, Xiamen University, Xiamen 361005, Fujian Province, P. R. China)
}

\begin{abstract}
Introducing surfactants including hexadecyltrimethylammonium bromide (CTAB), macrogol 6000 (PEG6000), and poly(ethylene glycol)-block-poly(propylene glycol)-block-poly(ethylene glycol) triblock copolymer (P123) into the refluxing aqueous crystal nucleus slurry yielded morphology-tuned microcrystalline $\gamma-\mathrm{MnO}_{2} . \gamma-\mathrm{MnO}_{2}$ and the influence of surfactant modification were investigated by X-ray diffraction (XRD), scanning electron microscopy (SEM), $\mathrm{N}_{2}$ adsorption (BET), thermogravimetry analysis (TGA), $\mathrm{O}_{2}$ temperature programmed desorption ( $\left.\mathrm{O}_{2}-\mathrm{TPD}\right)$, and temperature programmed $\mathrm{H}_{2}$ reduction $\left(\mathrm{H}_{2}-\mathrm{TPR}\right)$. Surfactants led to differences in $\gamma-\mathrm{MnO}_{2}$ morphology, surface area, oxygen desorption behavior and reducibility. The effect of reflux time on catalyst morphology is discussed. The catalytic performance of $\gamma-\mathrm{MnO}_{2}$ during the solvent-free atmospheric oxidation of toluene was evaluated. PEG6000 modified $\gamma-\mathrm{MnO}_{2}$ exhibited the highest catalytic activity judging by surface area because of a greater mixed valency and more anion vacancies. The greatest mass specific activity was obtained for $\mathrm{P} 123$ modified $\gamma-\mathrm{MnO}_{2}$ with the largest surface area. Optimized reaction conditions yielded an $18.1 \%$ toluene conversion, and 87.4 and $73.2 \%$ total selectivity and selectivity for benzoic acid, respectively.
\end{abstract}

Key Words: $\quad y-\mathrm{MnO}_{2} ; \quad$ Selective oxidation of toluene; Morphology tuning; Oxygen species; Surfactant

Received: September 20, 2012; Revised: January 2, 2013; Published on Web: January 4, 2013.

"Corresponding authors. YANG Le-Fu, Email: 1fyang@xmu.edu.cn; Tel: +86-592-2186291. FANG Wei-Ping, Email: wpfang@xmu.edu.cn; Tel: +86-592-2186291.

The project was supported by the National Key Basic Research Program of China (973) (2010CB226903).

国家重点基础研究发展规划项目(973) (2010CB226903)资助

(C) Editorial office of Acta Physico-Chimica Sinica 


\section{1 引言}

烃类选择性氧化在石油化工和精细化工中占 有极其重要的地位. 据统计, 催化过程生产的各类 有机化学品中, 选择性氧化产品约占 $25 \%$ 以上. ${ }^{1}$ 甲 苯的选择氧化产物苯甲醛与苯甲酸均为重要的有 机化工原料及中间体, 在染料、香料、食品防腐剂、 医药、塑料、合成纤维等领域有着广泛的应用, 因而 该氧化过程受到更大的关注. 目前工业上多以钴盐 或锰盐均相催化液相甲苯直接空气氧化来制备苯 甲酸, 副产品为苯甲醛. 虽然此方法存在着能耗高、 催化剂回收困难等技术短板, ${ }^{3}$ 但是甲苯液相直接空 气氧化法反应条件温和, 副产物可回收且具有很高 的经济价值, 加之使用空气或者氧气作为氧化剂的 成本低廉且环境友好, 因此深入探讨甲苯液相直接 氧化过程, 提高催化效率, 具有重要的理论意义和 实际应用价值.

在早期的甲苯液相直接氧化反应研究中, 溴化 物作为不可或缺的助剂存在于反应体系, ${ }^{4-6}$ 但在反 应温度下溴化物会加速设备的腐蚀, 而含溴废水也 将造成环境危害. 在此后的无溴反应体系中, 虽然 贵金属及过渡金属氧化物催化剂均已表现出较高 的催化活性, ${ }^{7-13}$ 但此类反应体系仍需要高温加压的 条件. 为降低甲苯在高温下的潜在操作风险 ${ }^{14,15}$ 并简 化反应装置, 以常压加热回流的方式进行甲苯选择 性氧化的设计逐渐受到重视. 由于甲苯侧链的 $\mathrm{C}-$ $\mathrm{H}$ 键为 $s p^{3}$ 杂化, 使得氧化反应需要较高的活化能, ${ }^{16}$ 常压下的氧化反应通常难以达到可观的转化效率 和收率, 所以催化剂的选择就尤为重要.

二氧化锰在比表面积、离子交换性、化学稳定 性及分子级的隧道空间等方面具有优异的性能, 其 独特的结构和理化性质使其在催化剂、 ${ }^{17-20}$ 电池、年-24 吸附剂 ${ }^{25}$ 等领域有着广泛的应用前景. 其中 $\gamma-\mathrm{MnO}_{2}$ 由 $\left[\mathrm{MnO}_{6}\right]$ 八面体单元堆积而成, 在一维方向上形成 具有不规则交替生长的软锰矿 $[1 \times 1]$ 隧道与斜方锰 矿 $[1 \times 2]$ 隧道晶胞, ${ }^{26}$ 该种无序交替生长结构导致晶 体中形成大量的缺陷、空位以及非理想配比, 使得 $\gamma-\mathrm{MnO}_{2}$ 呈现出独特的吸附活化特性, ${ }^{27}$ 是最常用的 一种二氧化锰材料, 也是为数不多被证明能够在常 压下促进甲苯液相直接氧化反应的催化剂之一 ${ }^{2}$

本文通过回流法合成 $\gamma-\mathrm{MnO}_{2}$ 催化剂, 引入 P123、PEG6000 和 CTAB 等表面活性剂以改变催化 剂的形貌、结构及氧物种反应特性, 并比较了它们 在常压回流反应条件下催化甲苯选择性氧化的活
性. 同时论文也考察了加热回流时间等制备参数对 催化剂形貌及性能的影响.

\section{2 实验部分}

\section{1 催化剂制备}

称取 $3.38 \mathrm{~g} \mathrm{MnSO}_{4} \cdot \mathrm{H}_{2} \mathrm{O}$ (分析纯), 用去离子水 配成 $100 \mathrm{~mL}$ 溶液, 加热至 $90^{\circ} \mathrm{C}$ 后, 加入 $4 \mathrm{~mL}$ $\mathrm{H}_{2} \mathrm{SO}_{4}$ (分析纯)酸化, 并继续搅拌 $10 \mathrm{~min}$. 向烧瓶中 逐滴滴加溶解有 $1.39 \mathrm{~g} \mathrm{KMnO}_{4}$ (分析纯)的去离子水 溶液 $100 \mathrm{~mL}$, 滴加完毕后加热回流 $30 \mathrm{~min}$, 加入不 同的表面活性剂, 之后继续回流一定的时间, 产物 经减压抽滤, 用去离子水洗涤直至滤液 $\mathrm{pH}$ 值接近 7 , 再用无水乙醇洗涤数次, 产物在真空干燥箱内 $60{ }^{\circ} \mathrm{C}$ 烘干过夜.

为考察表面活性剂的形貌控制效果, 制备了空 白样品以及分别加入表面活性剂 $0.109 \mathrm{~g}$ 十六烷基 三甲基溴化铵 $\left(\mathrm{CTAB}, 3 \times 10^{-4} \mathrm{~mol}\right.$, 厦门市先端科技 有限公司, 分析纯)、0.200 $\mathrm{g}$ 聚乙二醇 6000 (PEG $6000,3 \times 10^{-5} \mathrm{~mol}$, 上海化学试剂采购供应站, 分析 纯) 以及 $0.174 \mathrm{~g}$ 聚环氧乙烷-聚环氧丙烷-聚环氧乙 烷三嵌段共聚物(P123, 3×10 $0^{-5} \mathrm{~mol}$, Aldrich, 99\%)进 行调变的四种催化剂, 制备过程加热回流的时间为 $24 \mathrm{~h}$, 并记为 $\mathrm{rf}-\mathrm{MnO}_{2} 、 \mathrm{rf}-\mathrm{MnO}_{2} / \mathrm{CTAB} 、 \mathrm{rf}-\mathrm{MnO}_{2} / \mathrm{PEG}$ 、 $\mathrm{rf}^{-\mathrm{MnO}_{2}} / \mathrm{P} 123, \mathrm{rf}$ 表示制备方法为回流法(refluxing).

\section{2 催化剂表征}

采用荷兰PANalytical 公司 X'pert Pro 型X射线 粉末衍射仪进行 $X$ 射线粉末衍射(XRD)测试, 主要 测试条件为扫描范围 $(2 \theta) 20^{\circ}-80^{\circ}$, 扫描速率 $15\left(^{\circ}\right)$. $\mathrm{min}^{-1}$, 扫描步长 $0.016\left(^{\circ}\right) \cdot \mathrm{step}^{-1}$, 激发光源为 $\mathrm{Cu} K_{\alpha}$ 靶 $(\lambda=0.15406 \mathrm{~nm})$, 管电压 $40 \mathrm{kV}$, 管电流 $30 \mathrm{~mA}$; 采 用日本日立公司 Hitachi S-4800 扫描电镜(SEM)测 定样品形貌, 发射电压 $10 \mathrm{eV}$, 加速电压 $15 \mathrm{kV}$; 比表 面测试(BET)在美国 Micromeritics 公司 Tristar 3020 型全自动物理化学吸附仪上进行, 样品在 $150^{\circ} \mathrm{C}$ 下 真空处理 $2 \mathrm{~h}$, 以氮气为吸附质, 液氮温度下进行吸 附. 热重测试(TGA)在德国 Netszch 公司 TG 209 F1 热重分析仪上进行, 测试温度范围在 30-900 ${ }^{\circ} \mathrm{C}$ 区 间 $\left(\mathrm{N}_{2}\right.$ 气氛 $)$, 升温速率为 $10^{\circ} \mathrm{C} \cdot \mathrm{min}^{-1}$.

$\mathrm{O}_{2}$ 程序升温脱附 $\left(\mathrm{O}_{2}-\mathrm{TPD}\right)$ : 准确称取 $100 \mathrm{mg}$ 样 品, 在 $\operatorname{Ar}\left(\right.$ 流速 $40 \mathrm{~mL} \cdot \mathrm{min}^{-1}$ ) 吹扫下以 $10^{\circ} \mathrm{C} \cdot \mathrm{min}^{-1}$ 的 速率从室温升至 $300^{\circ} \mathrm{C}$, 恒温 $1 \mathrm{~h}$, 以对表面进行清 洁. 降至室温后切换成 $\mathrm{O}_{2}\left(\right.$ 流速 $20 \mathrm{~mL} \cdot \mathrm{min}^{-1}$ )进行预 吸附, 保持 $1 \mathrm{~h}$ 后切换为 $\operatorname{Ar}\left(\right.$ 流速 $20 \mathrm{~mL} \cdot \mathrm{min}^{-1}$ ) 吹扫 
至基线平稳后, 开始程序升温脱附, 以速率 $10{ }^{\circ} \mathrm{C}$. $\mathrm{min}^{-1}$ 升温至 $900{ }^{\circ} \mathrm{C}$, 以瑞士 Balzers 公司 Omnistar QMS200 四极质谱检测并记录 $\mathrm{O}_{2}(m / z=32)$ 信号得 $\mathrm{O}_{2}$ 脱附曲线, 同时检测 $m / z=40 、 m / z=28$ 等作为参考基线.

$\mathrm{H}_{2}$ 程序升温还原 $\left(\mathrm{H}_{2}-\mathrm{TPR}\right)$ : 取 $50 \mathrm{mg}$ 样品装于 样品管, 在 $\operatorname{Ar}\left(\right.$ 流速 $\left.20 \mathrm{~mL} \cdot \mathrm{min}^{-1}\right)$ 气氛下, 从室温升 至 $300{ }^{\circ} \mathrm{C}$, 稳定 $1 \mathrm{~h}$ 后降至室温, 切入 $5 \% \mathrm{H}_{2}-95 \% \mathrm{Ar}$ 混合气(流速 $40 \mathrm{~mL} \cdot \mathrm{min}^{-1}$ )吹扫至基线平稳; 然后以 $10{ }^{\circ} \mathrm{C} \cdot \mathrm{min}^{-1}$ 速率程序升温至 $700{ }^{\circ} \mathrm{C}$, 利用上海海欣 GC-950气相色谱仪测定并记录尾气中 $\mathrm{H}_{2}$ 浓度的变 化得还原曲线, 以同样测试条件下 $\mathrm{CuO}$ 的 $\mathrm{H}_{2}-\mathrm{TPR}$ 峰面积作为耗氢量基准, 对 $\mathrm{MnO}_{2}$ 系列样品的还原 峰面积进行定量校正.

\section{3 甲苯液相直接氧化反应催化性能评价}

取 $140 \mathrm{mg}$ 催化剂在 $120^{\circ} \mathrm{C}$ 空气气氛下, 预处理 $5 \mathrm{~h}$, 再与 $20 \mathrm{~mL}$ 甲苯(分析纯)及 $705 \mathrm{mg} A I B N$ (偶氮 二异丁腈, $0.5 \%$ (摩尔分数), 上海试四赫维化工有限 公司, 化学纯) 在三颈烧瓶内超声混合, 并向反应器 中通入 $\mathrm{O}_{2}\left(10 \mathrm{~mL} \cdot \mathrm{min}^{-1}\right)$, 于 $110{ }^{\circ} \mathrm{C}$ 油浴下以 $600 \mathrm{r}$. $\mathrm{min}^{-1}$ 搅拌回流 $20 \mathrm{~h}$. 反应结束后, 冷却至室温, 产物 用丙酮溶解并用 $100 \mathrm{~mL}$ 容量瓶定容, 经配有 HPINNOWax 毛细柱的岛津 GC-2014C 气相色谱仪分 离后, 以氢火焰离子化检测器(FID)分析组成.

\section{3 结果与讨论}

\section{1 表面活性剂改性 $\boldsymbol{\gamma}-\mathrm{MnO}_{2}$ 的物相及形貌表征}

表面活性剂在液相合成晶体的形貌控制以及 表面结构调变的功能一直备受关注. ${ }^{28-31}$ 而本研究使 用的归中反应原料 $\mathrm{KMnO}_{4}$ 的氧化性过强, 会与大多 数表面活性剂发生反应而造成改性失效, 并形成锰 氧化物. 因此, 我们选择了在成核段之后, 归中体系 氧化性降低的前提下, 加入表面活性剂以对晶体生 长段进行调控.

从图 1 的四种锰氧化物催化剂的 XRD 表征结 果可以看出, 归中反应能够在成核段决定产物的晶 相, 四种催化剂均在 $2 \theta=36.3^{\circ}, 41.5^{\circ}, 54.9^{\circ}, 65.4^{\circ}$ 左 右出现了主要的衍射峰, 并在 $2 \theta=22.6^{\circ}$ 附近也出现 了一个宽峰. 这些衍射峰均为 $\gamma-\mathrm{MnO}_{2}$ (JCPDS 14$644)$ 的特征峰, 其中 $2 \theta=22.6^{\circ}, 36.3^{\circ}, 41.5^{\circ}, 54.9^{\circ}$, $65.4^{\circ}$ 分别对应于 $\gamma-\mathrm{MnO}_{2}$ 的(120)、(131)、(300)、(160)、 (003) 晶面. ${ }^{26,32}$

在对比(003)晶面的衍射峰位可以发现, 表面活 性剂的添加导致了此衍射峰位向低角度发生了偏

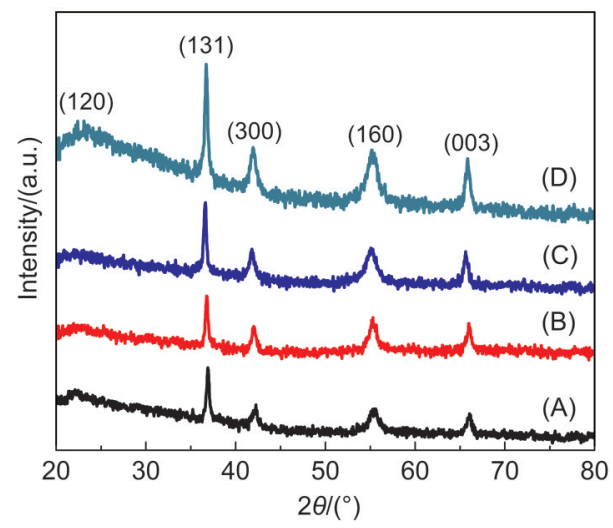

图 1 添加不同表面活性剂制得 $\gamma-\mathrm{MnO}_{2}$ 的 XRD 谱图

Fig.1 XRD patterns of $\gamma-\mathrm{MnO}_{2}$ by addition of different surfactants

(A) rf- $\mathrm{MnO}_{2}$, (B) rf- $\mathrm{MnO}_{2} / \mathrm{CTAB}$ (hexadecyltrimethylammonium bromide); (C) rf- $\mathrm{MnO}_{2} / \mathrm{PEG}$ (macrogol 6000), (D) rf- $\mathrm{MnO}_{2} / \mathrm{P} 123$ (poly(ethylene glycol)-block-poly(propylene glycol)-block-poly (ethylene glycol) triblock copolymer); the prefix rf- denotes the sample prepared by refluxing method.

移, 且偏移程度与表面活性剂种类有关. 这种晶格 堆垛方式导致的畸变, 应源于表面活性剂对 $\gamma-\mathrm{MnO}_{2}$ 晶体生长阶段结合水在晶格中的分布造成的影响, 早在 20 世纪 70 年代 Turner和 Buseck $\mathrm{k}^{33}$ 在验证 $\gamma-\mathrm{MnO}_{2}$ 的 De Wolff模型时就发现, 某些种类 $\gamma-\mathrm{MnO}_{2}$ 晶体中 有较多结合水的存在. Rüetschi ${ }^{34}$ 由此提出了阳离子 空位晶体结构模型, 认为 $\gamma-\mathrm{MnO}_{2}$ 的结合水是以 $\mathrm{OH}^{-}$ 的形式存在的, 由于 $\mathrm{OH}^{-}$与 $\mathrm{O}^{2-}$ 的半径比较接近, 因 此 $\mathrm{OH}^{-}$可能会取代晶格氧形成晶胞, 因而大量结合 水的存在会造成晶格畸变, 从而导致了 XRD 高角度 出峰偏移的现象. 同时较多水的存在也更容易导致 $\gamma-\mathrm{MnO}_{2}$ 晶胞生长的无序, 使得 XRD 的衍射峰弥散. 此外, 由 XRD 结果可见, $\mathrm{rf}-\mathrm{MnO}_{2} / \mathrm{P} 123$ 具有明显高于 其他三者的峰强度. P123具有聚乙二醇-聚丙二醇-聚 乙二醇三嵌段结构, 与合成六方晶系的介孔分子篎 SBA-15 的模板机制类似, P123 在水溶液中常自发形 成多分子聚集的胶束棒状, 并与水合物种相互作用, 在水解与缩聚的过程中, 通过表面活性剂与水合物 种之间的相互组合促进形成稳定的六方结构.

从图 2 的 $\mathrm{SEM}$ 表征结果来看, 与 $\beta-\mathrm{MnO}_{2}$ 纳米棒 微晶的形成机制类似, ${ }^{35}$ 在 $\mathrm{MnO}_{2}$ 晶核出现后, 即采 取柱状堆积的方式形成沿 $c$ 轴的优先生长, 而由于 在 $\gamma-\mathrm{MnO}_{2}$ 表面的缺陷位较多, 在没有表面活性剂的 干预下, $\mathrm{rf}-\mathrm{MnO}_{2}$ 倾向于形成针状物的海胆型聚集 体. 在晶体成长过程中, 加入的表面活性剂会覆盖 在生成的锰氧化物晶核表面, 表面活性剂的极性差 

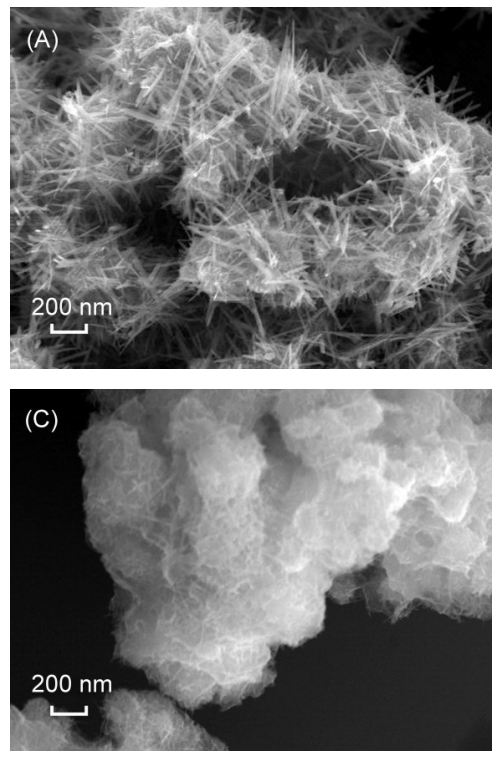

图 2 添加不同表面活性剂制得 $\gamma-\mathrm{MnO}_{2}$ 的 SEM 图片

Fig.2 SEM images of $\boldsymbol{\gamma}$ - $\mathrm{MnO}_{2}$ by addition of different surfactants

(A) rf- $\mathrm{MnO}_{2}$; (B) rf- $\mathrm{MnO}_{2} / \mathrm{CTAB}$; (C) rf-MnO $/$ /PEG; (D) rf- $\mathrm{MnO}_{2} / \mathrm{P} 123$

异对晶核的堆垛方式, 即晶体的继续生长将起到导 向作用. CTAB 由于基团中烷基长链的疏水性, 使其 吸附于晶核并包裹形成疏水层, 防止颗粒发生团 聚, 但在陈化过程中疏水膜之间的相互融合, 最终 会导致 rf- $\mathrm{MnO}_{2} / \mathrm{CTAB}$ 颗粒长大, 呈直径约 200$300 \mathrm{~nm}$ 的实心球状. P123 和 PEG6000 均为醇类高 聚物, 具有较好亲水性, 但相比之下, 线性长链结构 的 PEG6000 极性较高, 且含有大量的氢键, 因此生成 的镇氧化物容易发生聚集而形成层状结构, 并导致 合成的锰氧化物含水量偏高. 三嵌段结构的 P123 极 性略低, 与高极性水分子的互溶性适中, 可在晶核生 长初期与极性表面快速接触形成吸附层, 降低了在 表面缺陷位置发生枝化生长的几率, 同时也防止锰 氧化物过度水化导致团聚, 因此生成的锰氧化物分 散性最好, $\mathrm{rf}-\mathrm{MnO}_{2} / \mathrm{P} 123$ 呈现为细小针状晶体组成 的蓬松小球. BET测试结果显示, 四种锰氧化物的比 表面积分别为 $72.4 、 47.4 、 55.5 、 128.7 \mathrm{~m}^{2} \cdot \mathrm{g}^{-1}$, 比表面 积的次序与它们形貌分析呈现的趋势基本一致.

\section{2 陈化过程对 $y-\mathrm{MnO}_{2}$ 晶化效果的影响}

$\gamma-\mathrm{MnO}_{2}$ 微晶是由包含大量缺陷与空位的一维隧 道晶胞堆垛而成, 因此晶化过程进行的深度将对微 晶的生长产生影响. 参考上述表面活性剂种类对微 晶形貌的调变效应, 从晶相成长的完整性及微晶的 团聚特征考虑, 我们针对 $\mathrm{rf}-\mathrm{MnO}_{2} / \mathrm{P} 123$ 样品的陈化 时间对微晶相组成及形貌的影响作用进行了考察.

通过比较在 $90^{\circ} \mathrm{C}, \mathrm{P} 123$ 表面修饰作用下, 陈化
时间分别为 $6 、 12 、 24 、 48 \mathrm{~h}$ 的四个样品的相分析结 果(图 3)可见: 回流时间为 $6 \mathrm{~h}$ 时, 在 $2 \theta=36.3^{\circ}, 41.5^{\circ}$, $54.9^{\circ}, 65.4^{\circ}$ 处出现明显的 $\gamma-\mathrm{MnO}_{2}$ 衍射特征峰, 但在 $2 \theta=22.6^{\circ}$ 处对应于 (120)晶面的衍射峰尚未出现; 陈 化时间延长至 $12 \mathrm{~h}$ 后, 原有衍射峰无明显增强, 但 $2 \theta=22.6^{\circ}$ 处的衍射峰开始显现; 当陈化时间达到 24 $\mathrm{h}$ 时, 各衍射峰强度均有明显增强; 值得注意的是, 当陈化持续进行 $48 \mathrm{~h}$ 后, 原有 $\gamma-\mathrm{MnO}_{2}$ 行射峰强度发 生急剧减弱, 而在衍射图谱中, 斜方锰矿 (JCPDS 42-1316)的特征峰开始显现.

由相分析结果可以推测: 由于纤维状 $\gamma-\mathrm{MnO}_{2}$ 微 晶生长的各向异性, 不同指数晶面的堆积速率有很

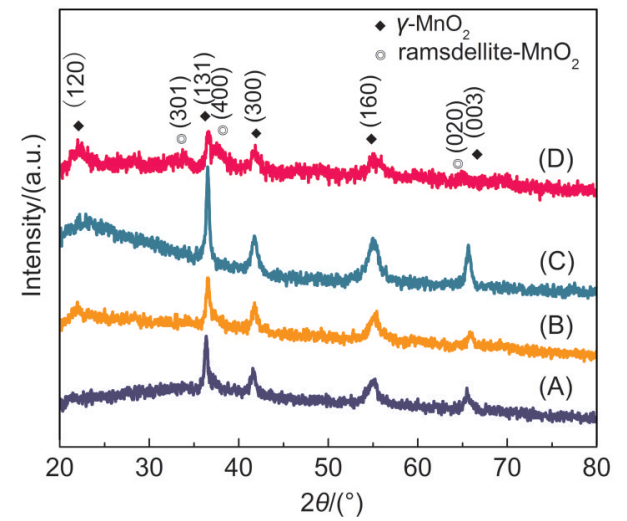

图 3 经不同加热回流时间处理的 $\mathrm{rf}-\mathrm{MnO}_{2} / \mathrm{P123}$ 的 XRD 谱图

Fig.3 XRD patterns of $\mathrm{rf}-\mathrm{MnO}_{2} / \mathrm{P123}$ with different refluxing time

(A) $6 \mathrm{~h}$; (B) $12 \mathrm{~h}$; (C) $24 \mathrm{~h}$; (D) $48 \mathrm{~h}$ 
大差异, (120)晶向上的堆积明显较慢,而在该方向 上的晶化进行至一定程度后, 微晶整体的规整度渐 趋完善并达到一个极限状态. 超过该状态后, 持续 陈化虽然强化了微晶在原 (120)晶向上的生长, 但受 到规整 $\gamma-\mathrm{MnO}_{2}$ 本征缺陷结构的电荷平衡作用的驱 动, 结合水逐渐以 $\mathrm{OH}^{-}$的形式取代晶格氧, 最终导致 斜方锰矿 $\left(\mathrm{MnO}_{2-x} \mathrm{OH}_{x}\right)$ 晶胞的形成. ${ }^{26}$

从 $\mathrm{SEM}$ (图 4)结果可看出 $\gamma-\mathrm{MnO}_{2}$ 微晶在不同生 长阶段的团聚倾向. 由于陈化 $6 \mathrm{~h}$ 时微晶表面尚存 在大量不饱和位, 从液相分离并干燥后, 纤维状 $\gamma-\mathrm{MnO}_{2}$ 发生了严重的团聚, 呈长有毛刺的块状结 构; $12 \mathrm{~h}$ 时, 表面晶化作用完成度较好, 不饱和位数 目下降, 催化剂团聚程度降低, 块状结构开始逐渐 分散, 并出现一些球状聚集体的雏形; 回流时间达 到 $24 \mathrm{~h}$ 时, 大部分产物呈现出细小的枝状组成的蓬 松的小球结构, 此时分散程度达到最高; 但继续增 加回流时间至 $48 \mathrm{~h}$ 后, 催化剂不仅相结构发生变 化, 而且晶格中 $\mathrm{OH}^{-}$的嵌入, 也会促进微晶颗粒间依 靠脱羟作用形成硬团聚, 使得颗粒发生了明显的增 大, 呈现出纳米棒团聚体的形貌. 这时 BET 表征结 果显示图 4(A-D)对应样品的比表面积分别为 48.1、 $60.7 、 128.7 、 75.5 \mathrm{~m}^{2} \cdot \mathrm{g}^{-1}$. 由此可见, 通过控制 $\gamma-\mathrm{MnO}_{2}$ 晶化进程, 能够进一步对微晶形貌和团聚程度实行 调节.

\section{$3.3 \mathrm{Y}-\mathrm{MnO}_{2}$ 晶格中氧物种的反应特性}

从不同表面活性剂修饰的 $\gamma-\mathrm{MnO}_{2}$ 样品的热失
重分析 (图 5)来看, 在经 $60{ }^{\circ} \mathrm{C}$ 真空干燥处理后, $\mathrm{CTAB}$ 表面活性剂所含溴离子在样品中有显著残 留, 且表现出强烈的还原能力, 在 $340{ }^{\circ} \mathrm{C}$ 之前即与 晶格氧发生氧化还原反应, 造成晶格氧的大量消 耗, 因此在 $\mathrm{O}_{2}-\mathrm{TPD}$ 图(图 6(B)) 中不再出现明显的脱 氧信号. 其余三种表面活性剂修饰样品, 结合 $\mathrm{O}_{2}$ $\mathrm{TPD}$ (图 6)表征结果, 在 $340^{\circ} \mathrm{C}$ 之前的失重台阶只能 归结为结合水的脱除, 而在 $350^{\circ} \mathrm{C}$ 至 $820^{\circ} \mathrm{C}$ 区间的 失重台阶则对应了 $\mathrm{MnO}_{2}$ 的脱氧还原, ${ }^{35} \gamma-\mathrm{MnO}_{2}$ 样品 的失重比率分别为 $9.8 \%\left(\mathrm{rf}-\mathrm{MnO}_{2}\right) 、 10.7 \%\left(\mathrm{rf}-\mathrm{MnO}_{2} /\right.$ $\mathrm{PEG})$ 和 9.7\%(rf- $\left.\mathrm{MnO}_{2} / \mathrm{P} 123\right)$, 接近从 $\mathrm{MnO}_{2}$ 到 $\mathrm{Mn}_{2} \mathrm{O}_{3}$ 的理论失重百分率 $9.2 \%$. 由上述分析可知, 脱氧过 程可涉及三个失重台阶, 对应温度区间分别为 $340-$ $500{ }^{\circ} \mathrm{C} 、 500-650{ }^{\circ} \mathrm{C}$ 以及 $650{ }^{\circ} \mathrm{C}$ 以上. 结合 $\mathrm{O}_{2}$ - TPD (图 6)表征结果, 可看出图 5 中 $340-500{ }^{\circ} \mathrm{C}$ 的失重台 阶可能包含脱水和脱氧两种过程: 未经表面活性剂 修饰的样品 $\mathrm{A}$, 因晶格规整度欠佳, 所以在该段发生 脱羟的同时伴随氧的脱除(图 6(A)); 而经 P123 修饰 的样品 $\mathrm{D}$, 该失重段几乎不含脱氧信号, 晶格氧在 $500-650{ }^{\circ} \mathrm{C}$ 温度段集中脱除. 另外, 热失重与 $\mathrm{O}_{2}-$ $\mathrm{TPD}$ 表征均证实了 $\mathrm{rf}-\mathrm{MnO}_{2} 、 \mathrm{rf}-\mathrm{MnO}_{2} / \mathrm{PEG}$ 甚至 rf$\mathrm{MnO}_{2} / \mathrm{CTAB}$ 在 $650{ }^{\circ} \mathrm{C}$ 以上存在高温脱氧段, 这部分 脱附氧对应着需要较高脱附活化能的 $\mathrm{Mn}^{3+} \rightarrow \mathrm{Mn}^{2+}$ 还原, 依据 Rüetschi 的 $\gamma-\mathrm{MnO}_{2}$ 阳离子空位晶体结构 模型, ${ }^{34}$ 在一维隧道结构中高价 $\mathrm{Mn}$ 离子的稳定性将 抑制该还原过程的进行, 因而 $\mathrm{rf}-\mathrm{MnO}_{2} / \mathrm{P} 123$ 能够得
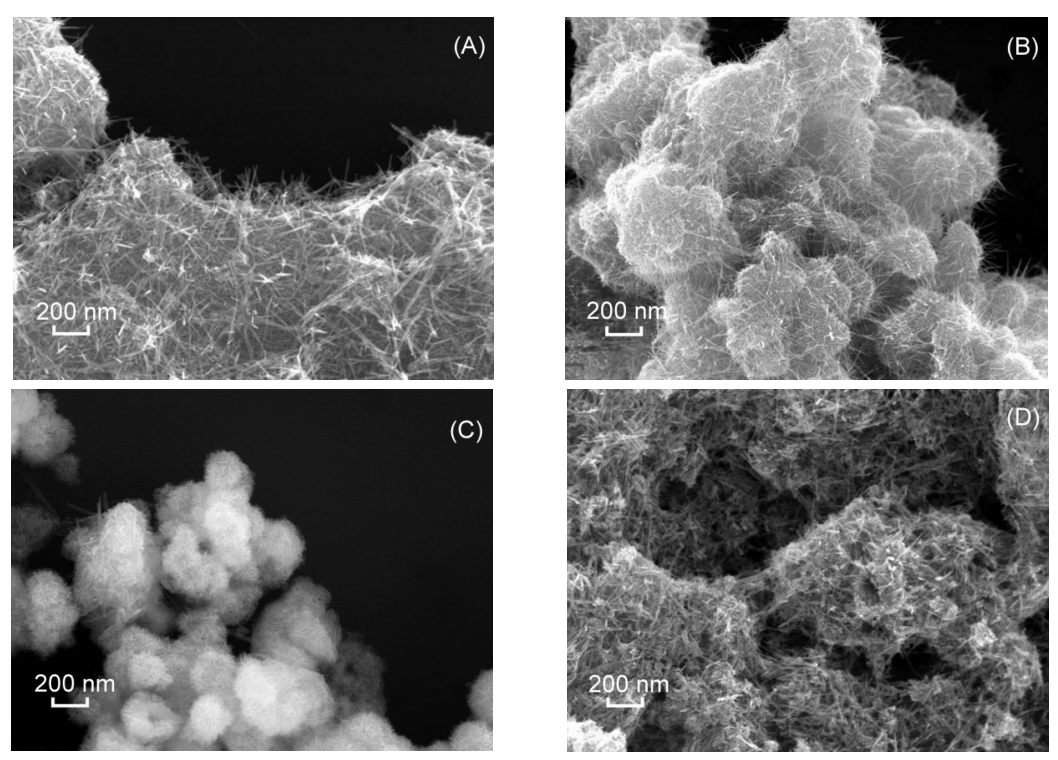

图 4 不同加热回流时间的 $\mathrm{rf}-\mathrm{MnO}_{2} / \mathrm{P123}$ 的 SEM 图片

Fig.4 SEM images of $\mathrm{rf}-\mathrm{MnO}_{2} / \mathrm{P123}$ with different refluxing time (A) $6 \mathrm{~h}$; (B) $12 \mathrm{~h}$; (C) $24 \mathrm{~h}$; (D) $48 \mathrm{~h}$ 

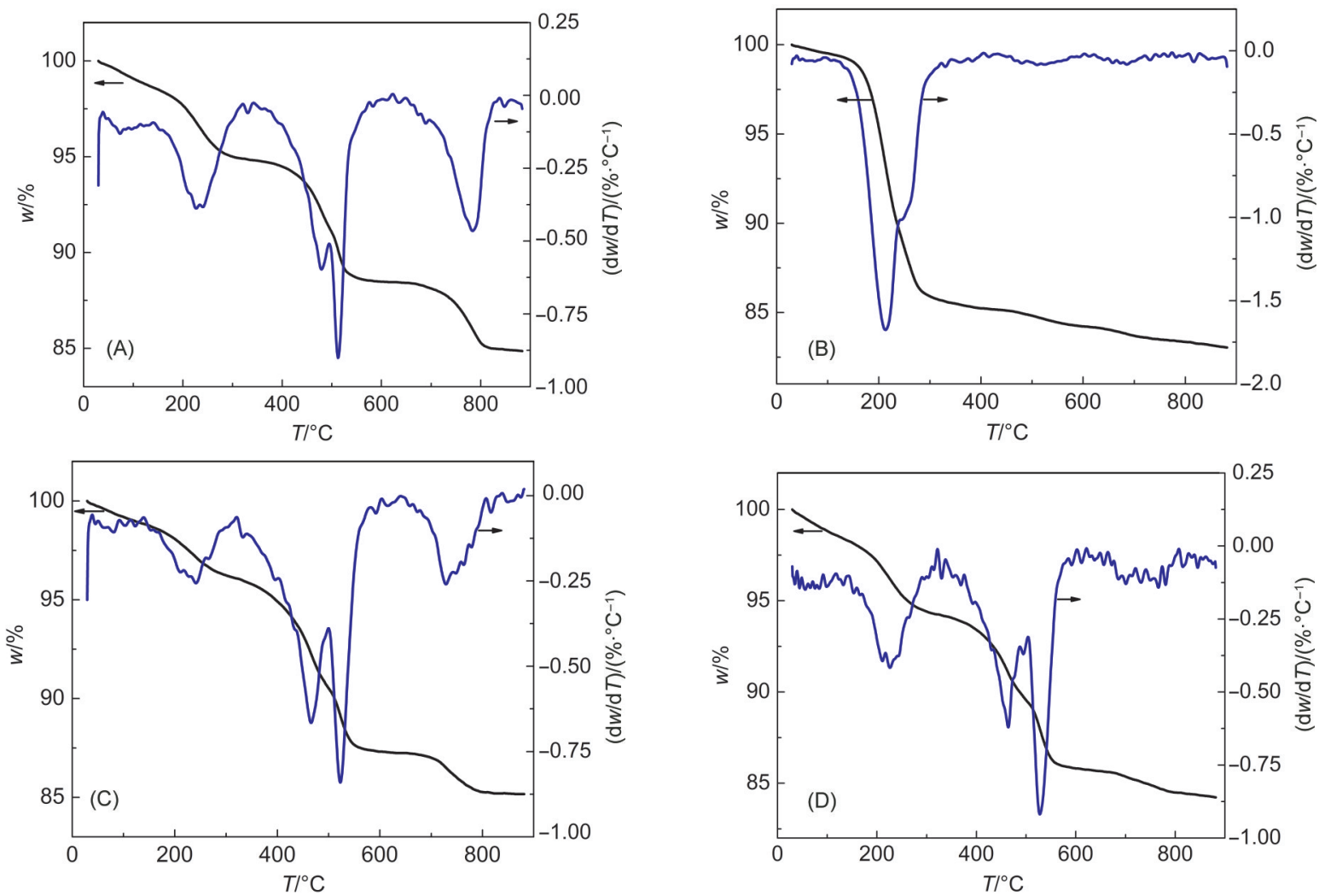

图 5 添加不同表面活性剂制得锰氧化物的 TG-DTG 谱图

Fig.5 TG-DTG analysis curves of $\boldsymbol{\gamma}-\mathrm{MnO}_{2}$ by addition of different surfactants

(A) $\mathrm{rf}-\mathrm{MnO}_{2}$; (B) rf-MnO $/ 2$ CTAB; (C) rf-MnO$/ 2 / \mathrm{PEG}$; (D) rf-MnO $2 / \mathrm{P} 123$

益于其良好的结晶性, 在高温段不发生明显的脱氧.

样品 $\mathrm{H}_{2}$-TPR(图 7)测试显示, 除 $\mathrm{rf}-\mathrm{MnO}_{2} / \mathrm{CTAB}$ 之外, 其它三个样品均在 $150-550^{\circ} \mathrm{C}$ 区间呈现出两 个明显的耗氢信号, 在 $150-330^{\circ} \mathrm{C}$ 区间的耗氢峰一 般被指认为 $\mathrm{MnO}_{2} \rightarrow \mathrm{Mn}_{3} \mathrm{O}_{4}$ 的还原进程, 而在 $330-550{ }^{\circ} \mathrm{C}$ 区间的耗氢信号则须对应更为深入的 $\mathrm{Mn}_{3} \mathrm{O}_{4} \rightarrow \mathrm{MnO}$ 还原进程. ${ }^{36}$ 在对耗氢量进行定量分析

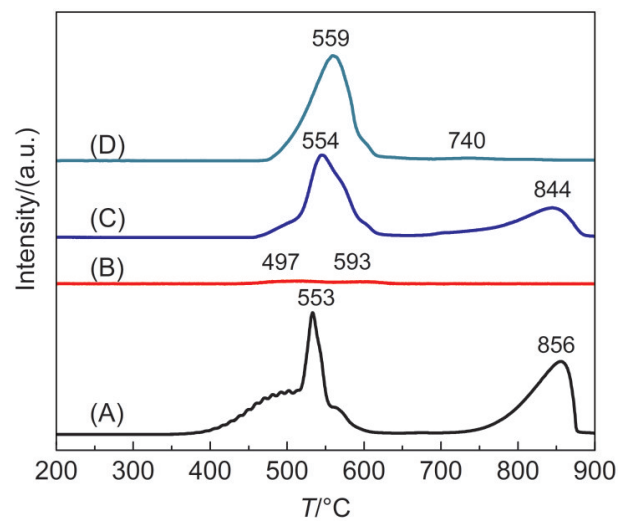

图 6 添加不同表面活性剂制得锰氧化物的 $\mathbf{O}_{2}$-TPD 谱图

Fig.6 $\mathrm{O}_{2}$-TPD profiles of $\gamma$ - $\mathrm{MnO}_{2}$ by addition of different surfactants

(A) $\mathrm{rf}-\mathrm{MnO}_{2}$; (B) rf- $\mathrm{MnO}_{2} / \mathrm{CTAB}$; (C) $\mathrm{rf}-\mathrm{MnO}_{2} / \mathrm{PEG}$; (D) $\mathrm{rf}-\mathrm{MnO}_{2} / \mathrm{P} 123$
(表 1)后发现, 未经氧预吸附处理的 $\gamma-\mathrm{MnO}_{2}$ 样品总耗 氢量均明显低于理论摩尔比值 $\left(n\left(\mathrm{H}_{2}\right) / n(\mathrm{Mn})=1\right)$, 说 明在 $\gamma-\mathrm{MnO}_{2}$ 样品中, 存在大量的阴离子空位及混合 价态 $\mathrm{Mn}(\mathrm{IV}) 、 \mathrm{Mn}(\mathrm{III})$, 其中样品 $\mathrm{rf}-\mathrm{MnO}_{2} / \mathrm{PEG}$ 中该 非计量特征尤为突出, 而对于结晶度最佳的 $\mathrm{rf}-\mathrm{MnO}_{2} / \mathrm{P} 123$ 样品, 耗氢量更为接近理论值, 且其高 温还原峰面积甚至超出 $\mathrm{Mn}_{3} \mathrm{O}_{4} \rightarrow \mathrm{MnO}$ 的理论耗氢

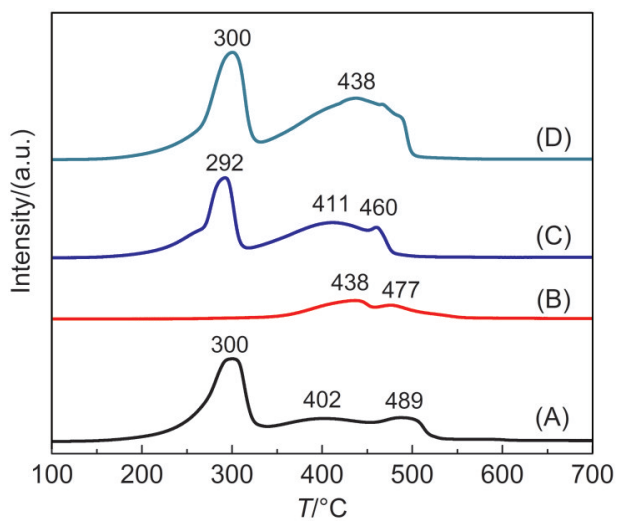

图 7 添加不同表面活性剂制得锰氧化物的 $\mathbf{H}_{2}$-TPR 谱图

Fig.7 $\mathrm{H}_{2}$-TPR profiles of $\gamma-\mathrm{MnO}_{2}$ by addition of different surfactants

(A) $\mathrm{rf}-\mathrm{MnO}_{2}$; (B) rf- $\mathrm{MnO}_{2} / \mathrm{CTAB}$; (C) rf- $\mathrm{MnO}_{2} / \mathrm{PEG}$; (D) $\mathrm{rf}-\mathrm{MnO}_{2} / \mathrm{P} 123$ 
表 1 各 $\gamma-\mathrm{MnO}_{2}$ 样品在不同还原段的耗氢 $\mathrm{H}_{2} / \mathrm{Mn}$ 摩尔比

Table 1 Staged hydrogen consumption of $\gamma-\mathrm{MnO}_{2}$ in terms of $\mathrm{H}_{2} / \mathrm{Mn}$ molar ratio

\begin{tabular}{lcc}
\hline \multirow{2}{*}{ Sample } & \multicolumn{2}{c}{$n\left(\mathrm{H}_{2}\right) / n(\mathrm{Mn})$} \\
\cline { 2 - 3 } & low temperature reduction & high temperature reduction \\
\hline $\mathrm{rf}-\mathrm{MnO}_{2}$ & 0.32 & 0.24 \\
$\mathrm{rf}-\mathrm{MnO}_{2} / \mathrm{CTAB}$ & - & 0.11 \\
$\mathrm{rf}-\mathrm{MnO}_{2} / \mathrm{PEG}$ & 0.21 & 0.24 \\
$\mathrm{rf}-\mathrm{MnO}_{2} / \mathrm{P} 123$ & 0.28 & 0.38 \\
\hline
\end{tabular}

表 2 不同表面活性剂对 $\gamma-\mathrm{MnO}_{2}$ 催化甲苯氧化性能的影响

Table 2 Effect of surfactant on catalytic performance of $\boldsymbol{\gamma}-\mathrm{MnO}_{2}$ for toluene oxidation

\begin{tabular}{|c|c|c|c|c|c|}
\hline \multirow{2}{*}{ Sample } & \multirow{2}{*}{ Conversion $/ \%$} & \multirow{2}{*}{$\frac{\text { Specific activity of surface area }}{\left(\mathrm{mol} \cdot \mathrm{m}^{-2} \cdot \mathrm{s}^{-1}\right)}$} & \multicolumn{3}{|c|}{ Selectivity/\% } \\
\hline & & & benzoic acid & benzaldehyde & benzyl alcohol \\
\hline $\mathrm{rf}-\mathrm{MnO}_{2}$ & 13.7 & $4.9 \times 10^{-9}$ & 65.4 & 11.4 & 4.2 \\
\hline rf- $\mathrm{MnO}_{2} / \mathrm{CTAB}$ & 6.9 & $3.8 \times 10^{-9}$ & 22.1 & 27.0 & 4.4 \\
\hline $\mathrm{rf}-\mathrm{MnO}_{2} / \mathrm{PEG}$ & 12.5 & $5.9 \times 10^{-9}$ & 68.7 & 13.6 & 5.0 \\
\hline $\mathrm{rf}-\mathrm{MnO}_{2} / \mathrm{P} 123$ & 18.1 & $3.8 \times 10^{-9}$ & 73.2 & 10.1 & 4.1 \\
\hline
\end{tabular}

reaction condition: $140 \mathrm{mg}$ catalysts was mixed in $20 \mathrm{~mL}$ toluene with $705 \mathrm{mg}$ AIBN (2,2'-azobis(cisobutyronitrile)). refluxing at $110{ }^{\circ} \mathrm{C}$ with $600 \mathrm{r} \cdot \mathrm{min}^{-1}$ stirring and $10 \mathrm{~mL} \cdot \mathrm{min}^{-1} \mathrm{O}_{2}$ flow

量 $\left(n\left(\mathrm{H}_{2}\right) / n(\mathrm{Mn})=0.33\right)$, 这应该是由于该 $\gamma-\mathrm{MnO}_{2}$ 晶格 经第一阶段还原后, 能够保留比 $\mathrm{Mn}_{3} \mathrm{O}_{4}$ 四方尖晶石 中更多的 $\mathrm{Mn}(\mathrm{III}) \mathrm{O}_{8}$ 八面体造成的.

\section{$3.4 \mathrm{y}-\mathrm{MnO}_{2}$ 的甲苯选择性氧化性能}

对于甲苯的氧化反应, 较大的甲苯分子(分子直 径约 $0.60 \mathrm{~nm})$ 将不能完全进入 $\gamma-\mathrm{MnO}_{2}$ 孔道 $([2 \times 2]$ 隧 道直径约 $0.46 \mathrm{~nm}$ ) 内, 故认为甲苯分子只能与位于 表面的氧物种发生氧化反应. ${ }^{35}$ 由此可以推测, 对于 $\gamma-\mathrm{MnO}_{2}$ 型催化剂来说: 一方面, 比表面积增大将会 获得更多的表面活性位点, 从而提高反应发生的几 率; 另一方面, 由于氧在甲苯中溶解度较高, 在液相 无溶剂反应条件下, 两种反应物的外扩散阻力都较 小, 所以在自由基引发剂的推动下, 提高溶解氧分 子的活化效率应是促进催化甲苯液相氧化反应的 关键步骤. ${ }^{2}$

表 2 中列出了不同表面活性剂改性催化剂的甲 苯液相直接氧化的活性测试结果. 在同样的测试条 件下 $\left(110{ }^{\circ} \mathrm{C}\right.$ 油浴及 $600 \mathrm{r} \cdot \mathrm{min}^{-1}$ 搅拌条件下回流 20 h), rf- $\mathrm{MnO}_{2} / \mathrm{CTAB}$ 因具有最小的比表面积, 以及高 价锰物种在制备过程中的消耗，使其只有不到 $6.9 \%$ 的转化率及 $53.5 \%$ 产物选择性. $\mathrm{rf}-\mathrm{MnO}_{2} / \mathrm{PEG}$ 虽然比 表面积小于 $\mathrm{rf}-\mathrm{MnO}_{2}$, 但两者的转化率和各产物收 率总体相近, 表面比活性超出后者接近 $20 \%$, 更超 出其它两个样品接近 $50 \%$. 该样品在前述氧化还原 表征中, 呈现出表面存有较多阴离子缺位及混合价 态的特征, 并在氧脱附测试中表现出较高的储氧能 力, 脱氧比率可达 $10.7 \%$ (质量分数), 此特点对于在
表面阴离子缺位上活化分子氧, 且同时在三相浆态 床反应的条件下提高供氧效率都十分有利. 而 $\mathrm{rf}-\mathrm{MnO}_{2} / \mathrm{P} 123$ 则由于其明显高于其它催化剂的比表 面积, 在较低的表面比活性的前提下, 也获得了 $18.1 \%$ 的转化率, 以及 $87.4 \%$ 的总选择性, 其中苯甲 酸的选择性达到 $73.2 \%$. 由此可见, 同时增大 $\gamma-\mathrm{MnO}_{2}$ 表面阴离子缺位数目并兼顾材料比表面积是提高 改型催化剂的甲苯液相直接氧化活性的关键所在.

\section{4 结 论}

在回流法制备过程中引入表面活性剂能够获 得在形貌上具有明显差异的 $\gamma-\mathrm{MnO}_{2}$ 微晶, P123 修饰 $\gamma-\mathrm{MnO}_{2}$ 微晶具有较好的结晶度与分散度. 陈化实验 证明, $\gamma-\mathrm{MnO}_{2}$ 晶格易受结合水嵌入影响而产生畸 变, 进而导致晶格坞塌而形成新的晶相.

$\gamma-\mathrm{MnO}_{2}$ 中氧的热稳定性随着结晶度提高而增 大, 同时其晶格中存有大量的阴离子空位及混合价 态, 该特征有助于分子氧在表面活化并增加储氧能 力, 这对增大甲苯液相直接氧化反应的催化效率非 常有利, 因此非计量特征突出的 $\mathrm{rf}-\mathrm{MnO}_{2} / \mathrm{PEG}$ 样品 表现出较高的表面比活性, 而增大 $\gamma-\mathrm{MnO}_{2}$ 的比表面 积对催化效率的促进也同样相当显著.

\section{References}

(1) Li, X. Q. Liquid-Phase Oxidation of Toluene over Manganese Oxides. Ph. D. Dissertation, Dalian Institute of Chemical Physics, Chinese Academy of Sciences, Dalian, 2006. [李晓 强. 锰氧化物对甲苯液相氧化反应的催化作用研究[D]. 大连: 
中国科学院大连化学物理研究所, 2006.]

(2) Jin, L.; Chen, C. H.; Crisostomo, V. M. B.; Xu, L. P.; Son, Y. C.; Suib, S. L. Appl. Catal. A 2009, 355, 169. doi: 10.1016/j.apcata. 2008.12.012

(3) Suresh, A. K.; Sharma, M. M.; Sridhar, T. Ind. Eng. Chem. Res. 2000, 39 (11), 3958. doi: 10.1021/ie0002733

(4) Holtz, H. D.; Gardner, L. E. Promoted Liquid Phase Oxidation of Alkyl Aromatic Compounds. U.S. Pat. 4088823, 1978.

(5) Kantem, M. L.; Choudary, B. M.; Sreekanth, P.; Rao, K. K.; Naik, K.; Kumar, T. P.; Khan, A. A. Process for the Production of Benzaldehyde by the Catalytic Liquid Phase Air Oxidation of Toluene. U.S. Pat. 6495726 B1, 2002.

(6) Kantem, M. L.; Sreekanth, P.; Rao, K. K.; Kumar, T. P.; Rao, B. P.; Choudary, B. M. Selective Liquid Phase Air Oxidation of Toluene Catalysed by Composite Catalytic System. U.S. Pat. 6743952 B2, 2004.

(7) Guo, C. C.; Liu, Q.; Wang, X. T.; Hu, H. Y. Appl. Catal. A 2005, 282, 55. doi: 10.1016/j.apcata.2004.11.045

(8) Ilyas, M.; Sadiq, M. Catal. Lett. 2009, 128 (3-4), 337. doi: 10.1007/s10562-008-9750-8

(9) Mohammad, S.; Mohammad, I. Chin. J. Chem. 2010, 28 (11), 2216. doi: 10.1002/cjoc.201090366

(10) Xue, M. W.; Chen, H.; Zhang, H. L.; Auroux, A.; Shen, J. Y. Appl. Catal. A 2010, 379 (1-2), 7. doi: 10.1016/j.apcata. 2010.02.023

(11) Xue, M. W.; Chen, H.; Ge, J. Z.; Shen, J. Y. Microporous Mesoporous Mat. 2010, 131 (1-3), 37. doi: 10.1016/ j.micromeso.2009.11.038

(12) Lv, J. G.; Shen, Y.; Peng, L. M.; Guo, X. F.; Ding, W. P. Chem. Commun. 2010, 46, 5909. doi: 10.1039/c0cc00777c

(13) Kesavan, L.; Tiruvalam, R.; Ab Rahim, M. H.; bin Saiman, M. I.; Enache, D. I.; Jenkins, R. L.; Dimitratos, N.; Lopez-Sanchez, J. A.; Taylor, S. H.; Knight, D. W.; Kiely, C. J.; Hutchings, G. J. Science 2011, 311, 195.

(14) Goethals, M.; Vanderstraeten, B.; Berghmans, J.; De Smedt, G.; Vliegen, S.; Van't Oost, E. J. Hazard. Mater. 1999, 70 (3), 93. doi: 10.1016/S0304-3894

(15) Zhao, W. F. J. Safety Sci. Tech. 2008, 4 (3), 157 . [赵文芳. 中国安全生产科学技术, 2008, 4 (3), 157.]

(16) Thomas, J. M.; Raja, R.; Sankar, G.; Bell, R. G. Nature 1999, 398 (6724), 227. doi: 10.1038/18417

(17) Perez-Benito, J. F. J. Phys. Chem. C 2009, 113 (36), 15982.

(18) Longo, A.; Liotta, L. F.; Di Carlo, G.; Giannici, F.; Venezia, A. M.; Martorana, A. Chem. Mater. 2010, 22, 3952. doi: 10.1021/ $\mathrm{cm} 100697 \mathrm{~b}$

(19) Fu, X. B.; Feng, J. Y.; Wang, H.; Ng, K. M. Nanotechnology 2009, 20, 375601. doi: 10.1088/0957-4484/20/37/375601
(20) Liao, W. P.; Yang, L.; Wang, F.; Hu, Y. F.; Sheng, Z. Y. Acta Chimica Sinica 2011, 69 (22), 2723. [廖伟平, 杨 柳, 王 飞, 胡宇峰, 盛重义. 化学学报, 2011, 69 (22), 2723.]

(21) Zhao, F. M.; Ma, C. A.; Chu, Y. Q.; Xu, Y. H. Acta Phys. -Chim. Sin. 2006, 22 (6), 716. [ 赵峰鸣, 马淳安, 褚有群, 徐颖华. 物理化学学报, 2006, 22 (6), 716.] doi: 10.3866/PKU. WHXB20060614

(22) Wang, Q.; Pan, J. Q.; Sun, Y. Z.; Wang, Z. H. J. Power Sources 2012, 199, 355. doi: 10.1016/j.jpowsour.2011.10.061

(23) Zhao, J. Z.; Tao, Z. L.; Liang, J.; Chen, J. Cryst. Growth Des. 2008, 8 (8), 2799. doi: 10.1021/cg701044b

(24) Wu, C. Z.; Xie, W.; Zhang, M.; Bai, L. F.; Yang, J. L.; Xie, Y. Chem. Eur. J. 2009, 15, 492. doi: 10.1002/chem.v15:2

(25) Qin, Q. D.; Wang, Q. Q.; Fu, D. F.; Ma, J. Chem. Eng. J. 2011, 172 (1), 68. doi: 10.1016/j.cej.2011.05.066

(26) Xia, X. Battery Bimonthly 2005, 35 (1), 27. [夏 熙. 电池, 2005, 35 (1), 27.]

(27) Hill, L. I.; Verbaere, A. J. Solid State Chem. 2004, 177, 4706. doi: 10.1016/j.jssc.2004.08.037

(28) Zhang, Z. R.; Pinnavaia, T. J. J. Am. Chem. Soc. 2002, 124, 12294. doi: 10.1021/ja0208299

(29) Parvulescu, V. I.; Bonnemann, H.; Parvulescu, V.; Endruschat, U.; Rufinska, A.; Lehmann, C. W.; Tesche, B.; Poncelet, G. Appl. Catal. $A$ 2001, 214 (2), 273. doi: 10.1016/S0926-860X(01) 00503-8

(30) Huang, Y.; Wu, J. H.; Huang, M. L.; Lin, J. M.; Huang, Y. F. Scientia Sinica Chimica 2011, 41 (1), 44 . [黄 毅, 吴季怀, 黄妙良, 林建明, 黄昀昉. 中国科学: 化学, 2011, 41 (1), 44.]

(31) Wang, Y. Y.; Zhao, C. W.; He, J. S.; Yan, Y.; Zhang, X. L.; Luan, Z. K. Chin. J. Inorg. Chem. 2012, 28 (2), 302. [王园园, 赵长 伟, 何劲松, 闰 勇, 张香兰, 奕兆坤. 无机化学学报, 2012, 28 (2), 302.]

(32) Fu, X. B.; Feng, J. Y.; Wang, H.; Ng, K. M. Mater. Res. Bull. 2010, 45, 1218. doi: 10.1016/j.materresbull.2010.05.014

(33) Turner, S.; Buseck, P. R. Science 1979, 203, 456. doi: 10.1126/ science.203.4379.456

(34) Rüetschi, P. J. Electrochem. Soc. 1984, 131 (12), 2737. doi: $10.1149 / 1.2115399$

(35) Ling, F.; Deng, L. J.; Chen, P.; Zhou, R. X.; Zheng, X. M. Journal of Zhejiang University (Science Edition) 2011, 38 (1), 73. [凌飞, 邓丽杰, 陈 平, 周仁贤, 郑小明. 浙江大学学 报(理学版), 2011, 38 (1), 73.]

(36) Ye, Q.; Huo, F. F.; Yan, L. N.; Wang, J. Cheng, S. Y.; Kang, T. F. Acta Phys. -Chim. Sin. 2011, 27 (12), 2872. [叶 青, 霍飞 飞, 闵立娜, 王 娟, 程水源, 康天放. 物理化学学报, 2011, 27 (12), 2872.] doi: 10.3866/PKU.WHXB20112872 